\title{
Die neugriechische Sprachforschung in den Jahren 1890 und $1891^{1}$ ).
}

I.

Nur sehr gering ist die Zahl der Gelehrten, welche, mit wissenschaftlicher Methode ausgerüstet, Forschungen auf dem Gebiet der neugriechischen Sprache betreiben. Wenn trotzdem die Anzahl der Abhandlungen, über welche ich im folgenden referiere, verhältnismässig gross ist, so rührt das davon her, dass die neugriech. Sprachforschung entweder von angrenzenden Wissensgebieten Aufschlüsse erhält, oder dass Dilettanten mit mehr oder weniger Geschick auf dem brach liegenden Felde sich tummeln, oft auch zu grösserem Nutzen der Wissenschaft in der Herbeischaffung von Material Dienste leisten.

Wir beginnen mit Arbeiten, die sich auf die Geschichte der neugriechischen Studien beziehen. Noch im Jahre 1889 hat uns W. Meyer eine Ausgabe einer der ältesten neugr. Grammatiken, der des Simon Portius (1638), bescheert. Psichari gab in einer Einleitung dazu biographische Erörterungen und glaubte aus linguistischen Gründen erweisen zu können, dass Simon Portius ein Kreter gewesen sei. Diese Frage hat für die Beurteilung der Grammatik des Simon Portius ziemliche Bedeutung: sie erhielt endgiltige Lösung dureh den Aufsatz von Legrand Contribution à la biographie de Simon Portius, Revue des Etudes grecques IV (1891) p. 74-81.

Portius stammt aus Trapezunt, dies ist das wichtigste Ergebnis der Abhandlung, die ausserdem einige weitere biographische und litterarhistorische Nachweise über Portius gibt.

Der grösste Geistesheros des modernen Griechenlands,

1) D. h. etwa bis Mitte 1891; einigemal ist über das Jahr 1890 zurückgegriffen worden, sei es um eine gewisse Kontinuitiat herzustellen, sei es um auf besonders Wichtiges aufmerksam $z u$ machen. Vollständigkeit der bibliographischen Angaben ist erstrebt, für WestEuropa hoffentlich auch erreicht. In bezug auf griech. Zeitschriften, Zeitungen und Bücher ist es bei dem Mangel einer Zentralisation des griech. Buchhandels ausserordentlich schwer, einen vollständigen Überblick über griech. Publikationen zu bekommen. Trotzdem hoffe ich, Wichtiges nicht übersehen zu haben. Finige Ungenauigkeiten von Zitaten bitte ich damit entschuldigen zu wollen, dass ich beim Niederschreiben meines Referats hin und wieder auf die Exzerpte angewiesen war, welche ich von Schriften angefertigt hatte, die mir seinerzeit vorlagen, jetzt aber nicht mehr zugänglich sind. Die hiesige Universitätsbibliothek hat (wie wohl die meisten deutschen Bibliotheken) nur einen geringen Bestand an Neograeca, so dass ich vielfach auf meine eigenen Erwerbungen angewiesen war, 
der Schöpfer der neugr. Schriftsprache, Adamantios Korais, hat in Thereianos einen sachverständigen und begeisterten Biographen gefunden:

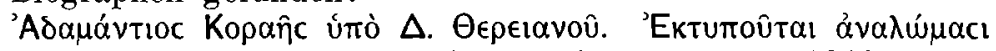

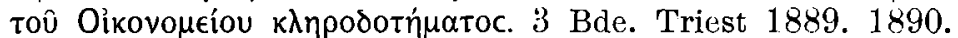

Rezensionen: 'Ectía 18. März 1890. Seibel in der Wochenschr. für klass. Philol. VIII Sp. 539 ff. Schenkl Zeitschr. für österreich. Gymnasien XLI 527-529. Neue philol. Rundschau 1891 p. 224. Tozer Academy 1891 No. 998. A. Wagener Bl. f. d. bayer. Gymnasialwesen XXVII (1891) p. 243250. Zimmerer Berl. phil. Wochenschr. XI No. 39 f.

An dieser Stelle ist vor allem der zweite Band hervorzuheben, worin des Korais Ansichten üßer die neugr. Sprache und seine Thätigkeit für eine neugriech. Schriftsprache eingehend dargestellt werden. Thereianos verfolgt im Anschluss daran die Entwicklung der sogenannten Sprachfrage bis auf unsere Tage.

Neugriechische Sprache und Literatur in Deutsch-

land ist skizziert in der Beilage der Allgem. Z(jitung 1890, 16. und 17. Dezember.

Von bibliographischen Zusammenstellungen übor neugricchische Dinge ist zu nennen der Bericht von Oberhumm e $\mathbf{r}$ übor griecl. Geographic in Bursians Jahresberichten Bd. 64 (1891), besonders die Abschnitte, welche die heutige Bevölkerung betreffen, nämlich p. 389-403, $407 \mathrm{ff}$., $411 \mathrm{ff}$. (Reisewerke), 439 (Name von Morea), 443 f. (Ethnographic). Bei dem Mangel an ausgedehnten und eingehenden Beobachtungen über neugriech. Sprache und Dialekte sind wir oft froh, in Werken heterogenen Inhalts einige sprachliche Notizen zu finden; es muss freilich auch betont werden, dass solche Notizen, die in Reiscwerken oder Aufsätzen ethnographischen Inlualts begegnen, meist sehr ungenau sind und scharfer Prüfung bedürfen.

Von grundlegender Bedeutung auch für die neugr. Sprachgeschichte ist die Ethnographie der Balkanhalbinsel, vor allem sofern sie die Frage von der Abstammung der heutigen Griechen, d. h. die Verwandtschaftsverhältnisse zwischen den alten Hellenen und den modernen Griechen, behandelt. Die neugr. Sprache zeigt zwar (um von anderm zu schweigen) klar, dass sie eine Fortentwicklung des Altgriechischen ist, und die These Fallmerayers lässt sich heutigentags nicht mehr aufrecht erhalten, aber trotzdem ist es wünschenswert, an der Hand genauer Statistik die heutige Verbreitung des griechischen Elements im Vergleich zu der im Altertum und derjenigen fremder Elemente auf einst griechischem Boden zu ermitteln und den Grad fremden Ein- 
flusses festzustellen. An dieser Aufgabe ist auch die Sprachforschung beteiligt; hier seien nur die speziell ethnographischen Arbeiten aufgezählt:

A bstammung der heutigen Griechen (ohne Autornamen), Zeitschr. f. Schulgeogr. VIII 340-342 (mir nicht zugänglich).

X e n opol Les Roumains et les Grecs. Revue de géogr. 1891 (mir nicht zugänglich.)

Oppel Zur Ethnographie der Balkanhalbinsel, Globus Bd.57 (1890) p. 76-79. (Ubersicht über die bisherigen Arbeiten; Griechen im heutigen Makedonien.)

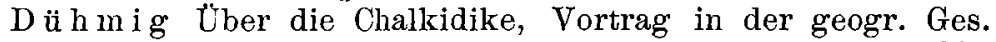
zu München. Cf. Verh. d. Ges. f. Erdk. z. Berlin 1891 p. 102. (Die Chalkidike durchaus griechisch, womit auch Oppel übereinstimmt.)

Über das griechische Element in Kleinasien belehrt uns in anthropologiseher Hinsicht

$\mathrm{Lu} \mathrm{s} \mathrm{chan} \mathrm{Reisen} \mathrm{in} \mathrm{Kleinasien,} \mathrm{Verhandl.} \mathrm{der} \mathrm{Gesellsch.} \mathrm{f}$. Erdkunde zu Berlin XV 47-60.

Ich hebe daraus hervor (p. 55 f.), dass nur auf den Inseln und an der Westküste in hohem Prozentsatz der altgriech. Typus vertreten ist, während die Griechen im Innern, sowie an der Süd- und Nordküstc physisch mit den Armeniern übereinstimmen; eine dritte Gruppe von Griechen mit semitischem Typus (Reste altsemitischer Kolonisation) beobachtete Luschan an der Südküste. Darüber vgl. auch denselben Gelehrten in seinem Aufsatz Die Tachtadschy und andere Überreste der alten Bevölkerung Lykicns, Archiv für Anthropologie XIX 31-5̃3. Auch unter den nichtgriech. Bewohnern Kleinasiens fand Luschan griechische Spuren.

$\mathrm{Ob}$ der Aufsatz von

Gh en a d i f f La Macédoine, Bull. de la Soc. belge de géogr. Nr. 6 (1891)

Angaben über die griech. Bevölkerung enthält, weiss ich nicht, da mir die Zeitschrift nicht zugänglich ist.

Von besonderem Werte und holier Zuverlässigkeit sind die Arbeiten Philippsons über die Ethnographie des Peloponnes. Einen kurzen Überblick gibt uns dieser Gelehrte in seinem Aufsatz

Besiedelung im Peloponnes, Verh. der Ges. f. Erdk. zu Berlin XV 442-4う5.

$\mathrm{Ph}$. betont den Mischcharakter der peloponnesischen Bevölkerung, von der bekanntlich die Albanesen bis jetzt noch der Hellenisierung entgangen sind. In sprachlicher Beziehung wird hervorgehoben, dass das peloponnesische Griechisch 
(abgesehen vom Tzakonischen) ohne bedeutende dialektische Unterschiede sei.

Ausführlicher beschäftigt sich $\mathrm{Ph}$. mit derselben Frage in Zur Ethnographie des Peloponnes, Petermanns Mitteilungen 1890 p. $1-11,33-41$.

In einem geschichtlichen Utberblick werden auf grund der neueren Forschungen über byzantinische Geschichte die Einwanderungen fremder Stämme (besonders der Slaven, dann auch der Franken, Osmanen und endlich Albanesen) besprochen. Nur die Albanesen haben sich, wie erwähnt, noch bis heute gehalten, obwohl ein allmähliches Zurücktreten deutlich konstatiert werden kann. Die heutige Bevölkerung des $\mathrm{Pe}$ loponnes enthält c. $12 \%(90000)$ Albanesen. Ich muss es mir versagen, die Details über deren Verbreitung wiederzugeben; Ph., der den Peloponnes mach allen Seiten durchwandert hat, gibt in Tabellen und einer Karte genaue Auskunft. Ich erwähne als charakteristisch, dass zwischen Griechisch und Albanesisch scharfe Grenzen bestehen, dass vor allem nicht dic Bildung einer Mischsprache $\mathrm{zu}$ beobachten ist. Über die Tzakonen und Maniaten s. unten. Im Norden des Peloponnes sitzen einige rumeliotische Nomaden, die vielleicht Reste der um 1709 eingewanderten 6000 Rumelioten sind

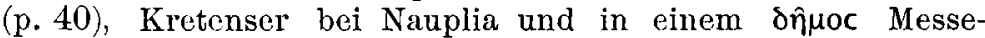
niens; andere Elemente (Zigeuner, Vlachen) kommen heute nicht mehr in betracht. Ph. gelangt zu dem Ergebnis, dass die heutigen Peloponnesier ein fast völlig hellenisiertes Mischvolk sind ${ }^{1}$ ).

Über die Albanesen im übrigen freien Griechenland erhalten wir von Philippson ebenfalls Auskunft in

Reise dureh Mittel- und Nordgriechenland, Zeitschr.

d. Ges. f. Erdk. zu Berlin, Bd. XXV (1890) p. 331-406, bezw. p. $402 \mathrm{f}$.

Das albanesische Element beträgt nach Ph. für ganz Griechenland 11,3\% (Euboea 40000, Nord-Andros 10000, Attika und Bocotien mit Megara 84000).

1) Die Resultate Philippsons haben manche Griechen unangenehm berührt. So hat ein gewisser Mitsopulos in einer populären naturwiss. Zeitschrift ( $\Pi_{\rho \circ \mu \eta} \theta \in u$ c, des genaueren Zitats erinnere ich mich nicht mehr) zwar mit grossem chauvinistischen Eifer, aber mit desto geringerer Wissenschattlichkeit Philippsons Forschungen herunterzusetzen versucht, freilich ohne Erfolg. Dem gegenüber muss lobend hervorgehoben werden, dass ein anderer Grieche, der tüchtigre Geograph Miliarakis, die Verdienste Philippsons um das Gebiet der ethnographischen Statistik voll und ganz anerkennt (in einer Rezension in dem nach einigen Nummern wieder eingegan-

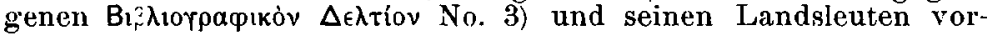
hält; statt müssiger Redereien ̈̈hnliche Untersuchungen anzustellen. 
In bezug auf Zuverlässigkeit der Beobachtung darf neben Philippson der schon erwähnte Grieche A. Miliarakis genannt werden. Leider kenne ich sein letztes Buch

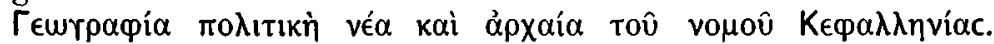
Athen 1890

nur aus der Besprechung in der 'Ectía vom 18. November 1890 ( $\pi \alpha \rho \alpha \dot{\rho} \tau \tau$.) und aus dem Referat von Partsch Petermanns Mitteil. 1891 (Literatur-Bericht p. 28). Darnach enthält es wertvolle Angaben über die Bevölkerung, über Orts- und Familiennamen. (In der Südostecke von Cefalonia wohnen Albanesen, die im 15. Jahrh. eingewandert sind; so erkläre sich das Vorkommen des Namens $\mu \pi \alpha ́ \lambda \tau \alpha$; doch macht Partsch darauf aufmerksam, dass der Name schon 1262 urkundlich sich finde).

Beiträge zur Volkskunde (Mythologie etc.) enthalten Tozer Islands of the Aegean. Oxford 1890 (Clarendon Press) und

Ros cher Studien zur griech. Mythologie, IV. Heft mit einem Anhang von Politis Uber die bei den Neugriechen vorhandenen Vorstellungen vom Monde.

Bekanntlich ist gerade die neugr. Volkskunde vorzüglich geeignet, don engen ethnischen Zusammenhang zwischen den alten Hellenen und den Neugriechen klar zu erweisen. Während Politis ein spezielles Gebiet behandelt, finden wir in dem Reisewerke von Tozer da und dort Notizen über griech. Aberglauben, Sitten und Gebräuche. Tozer gehört zu den wenigen Philologen, die bei ilrren topographischen und antiquarischen Studien auch das moderne Griechenland und seine Bevölkerung gebührend berücksichtigen. 'T.'s Reisewerk bietet eine geschickte Übersicht dessen, was bis jetzt über die von ihm bereisten Gebicte (Geschichte, Land und Leute) bekannt ist. Auch der Sprachforscher findet hin und wieder zwar nicht ausgedehnte aber doch schätzenswerte Angaben. Darüber weiter unten. Von Besprechungen des Werkes kenne ich Partsch Petermanns Mitteil. 1890 Lit.-Ber. No. 2467 und Paton The Classical Review V (1891) p. 237-238.

Ich muss es mir versagen, hier weiteres zur Volkskunde

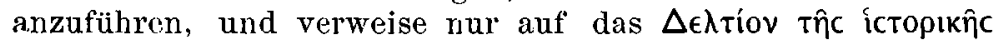

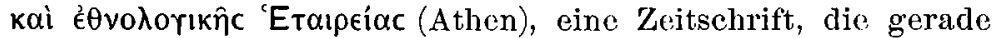
auch diese Seite der neugriech. Philologie zu fördern verspricht.

II.

In der Publikation neuer mittelgriechischer Texte haben die letzten Jahre nur weniges geleistct. Einen kurzen Bericht über mgr. Handschriftenschätze der Konstanti- 
nopler Bibliothek vom heil. Grab gibt Psichari in seinem weiter unten zu besprechenden Rapport p. 29. Ein grösserer Text wurde von Legrand publiziert als Bd. V. der Bibliothèque grecque vulgaire:

La guerre de Troje, Poème du XIV e siècle en vers octosyllabes par Constantin Hermoniacos. Publié par E. Legrand. Paris 1890. XIV $478 \mathrm{~S}$.

Rez. von Psichari Revue critique 1891 (I) p. 28-30.

In der Vorrede wird der Sprachcharakter der Dichtung ganz vom Standpunkt Psicharis beurteilt; das Gedicht gebe die gleichzeitige Sprache von Epirus wieder. Der Text. selbst ist nach L. eine Bearbeitung von Tzetzes Allegoriae Iliadis. 3 Handschriften aus dem 15. Jahrhundert standen dem Herausgeber zu Gebote (2 Pariser und eine aus Leyden). Wertvoll ist der Index (p. $459 \mathrm{ff}$.), der von Legrand hinzugefügt wurde und der alle Spracheigenheiten des "Dichters" umfasst.

7 kleinere Gedichte des Prodromos veröffentlichte gleichfalls

Le g r a n d Poésiés inédites de Théodore Prodrome, publiées d'après la copie d'Alphonse l'athénien, Revue des ćtudes grecques IV $70-73$.

Hohes sprachgeschichtliches Interesse beansprucht eine vulgärgriechische Ubersetzung des Pentateuch, die von einem Juden Konstantinopels verfertigt und im Jahr 1547 in hebräischen Lettern gedruckt wurde. İber das seltene, in Paris befindliche Buch handelt

Belleli Deux versions faites à Constantinople au seizième siècle. Paris 1890 (16 S.),

eine Schrift, dic ich aus der Besprechung in der 'Ectía vom 19. August 1890 kenne. Um die rein litterarhistorische Seite hier zu übergehen. hebe ich die Bemerkung hervor, dass der Text für die Kenntnis der damals in Konstantinopel gesprochenen Volkssprache eine gute Quelle ist. Der Kritiker in der 'Ectía bezweifelt dies, weshalb Belleli in einer Entgegnung in der "Ectía vom 26. August 1890 ( $\pi \alpha \rho$.) betont, "ơtr

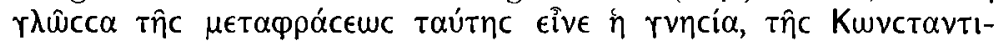

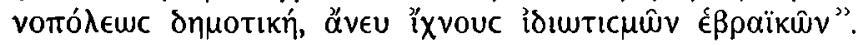

Eine Probe des interessanten Textes giebt Belleli in der Revue des Etudes grecques III 289-308. In der Einleitung dazu finden wir einige Notizen über die Sprache der Übersetzung. Auf diese selbst folgt ein kurzer (grammatischer) Kommentar. Was übrigens die dem Herausgeber

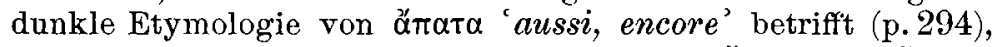

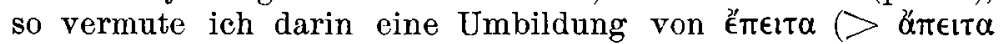

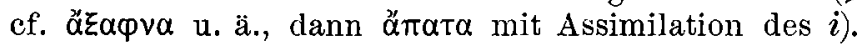


Nur in loser Beziehung zur mittelgriech. Philologie steht der Aufsatz von

J. Psichari Le Roman de Florimont. Contribution à l'histoire littéraire. Etude des mots grecs dans ce roman. In den Etudes Romanes dédiées à Gaston Paris. Paris 1891 p. $507-550^{1}$ ).

Rez. von Suchier, Lit.-Bl. f. germ. u. rom. Philol. 1891 Sp. $273 \mathrm{ff}$.

Ps. beschäftigt sich vor allem mit den in den Text eingestreuten griechischen Wörtern, die er aus ihrer verstümmelten Form (in lateinischen Lettern) wieder herzustellen sucht; hinsichtlich des sprachgeschichtlichen Wertes dieser Wörter kommt Ps. zu dem Ergebnis "Les formes grecques du Florimont n'ont aucun intérêt en elles-mêmes. Elles ne nous apprennent rien sur la grammaire historique du grec au moyen âge". Aber diese griech. Formen geben in ihrer Verstünmmelung wichtige Aufschlüsse über die Textgeschichte des altfranz. Romans, ein Problem, das jedoch mehr für die Romanisten als für uns Interesse hat.

Für alle Fragen über mgr. Texte, ihre Geschichte und Sprache sowie die einschlägige Bibliographie giebt ein Werk treffliche Belehrung, das für das ganze Gebiet einen festen Grund und Boden geschaffen hat:

Krumbacher Geschichte der byzantinischen Literatur (J. v. Müller, Handbuch der klass. Altertumswiss. IX 1). Münehen 1891.

Der Wert des Buches ist so allgemein anerkannt, dass es genügt, hier auf die Rezensionen zu verweisen: G. Meyer, Beilage der Allgem. Zeitung 1890 No. 297. Usp. . . iy Lit. Centralbl. 1891 Sp. 240-244. Weyman Histor. Jahrbuch XII 79--86 (mit bibliographischen Nachträgen). Merkle Studien und Mitteilungen aus dem Benediktiner- und Cistercienser-Orden XII 1. Oster Neue philol. Rundschan 1891 p. 204 208. Gelzer Berl. philol. Wochensehr. XI No. 27 und 28. Draeseke Theolog. Literatur-Zeitung 1891 p. 329-334.

Aus dem Gesammtgebiet der Lexikographie nenne ich zunächst, wenn auch einem etwas früheren Zeitraum als dem zu besprechenden angehörig, die Neuauflage des monumentalen Werkes von

Sophocles, A Greek Lexicon of the Roman and Byzantine periods, New York und Leipzig 1888.

Das Lexikon umfasst den spätgr. und mgr. Wortschatz (bis auf 1100); als Einleitung ist eine kurze Grammatik des

1) Mir liegt durch die Güte des Herrn Verfassers ein Separatabzug ror. 
Vulgärgriechischen in geschichtlicher Entwicklung vorausgeschickt.

Einige lexikalische Anmerkungen zu dem Werke findet man in der Rezension von Zenos The Classical Review IV (1890) p. $41-44$.

Fin anderes älteres Werk stupenden Fleisses, das Glossarium ad scriptores mediae et infimae graecitatis von $\mathrm{Du}$ cange ist durch einen unveränderten Neudruck (Breslau 1891, Koebner, 2 Bde.) wieder leichter zugänglich gemacht worden, wenn auch der Preis immer noch ein ziemlich hoher ist.

Der neugriechischen Etymologie werden sehr grosse Dienste geleistet durch

G. Meyer Etymologisches Wörterbuch der albanesischen Sprache. Strassburg, Trübner 1891.

Indem Meyer den verschlungenen Pfaden albanesischer Lexikographie nachgeht, gibt er uns wertvolle Aufschlüsse über die Etymologie und Lexikographie der Sprachen der Balkanhalbinsel und schafft Klarheit in den bunten Wirrwar von Entlehnungen. Da gerade die Beziehungen zwischen AIbanesisch und Neugriechisch besonders enge sind, so tritt das Gricchische nicht wenig hervor. Ein Wortverzcichnis (p. 505 ff.) orientiert uns rasch darüber.

Ein spezielles Gebiet der neugriechischen Lexikographie behandelt

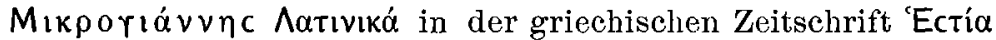
1891 No. 30 und 31.

M. ist Schüler von Psichari; er schreibt in neugriechischer Volkssprache. In der Form eines Dialogs werden die wichtigsten lateinischen Lehnwörter des Neugriechischen, ihre Lautgesetze und die Kriterien ihrer Scheidung von den romanischen Lehnwörtern besprochen.

Von etymologischen Einzelbeiträgen sind zu nennen:

Hesseling Istambol, Revue des Etudes grecques III 189196. (Entstehung und türkische Umbildung des Namens.

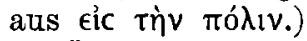

Uber den Namen 'Morea' vgl. die Zusammenstellungen von Etymologien bei

Gregorovius Geschichte von Athen I 309 f. und

Oberhummer in dem schon genannten Bericht p. 439 .

Ferner zur Etymologie geographischer Namen:

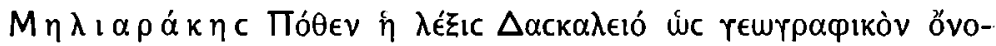
$\mu \alpha$; ${ }^{c}$ Ectía 1890 (I) p. 43.

(Der öfters für kleine Felseneilande begegnende Name $\triangle \alpha c k \alpha \lambda \epsilon 1$ ó wird als volksetymologische Umgestaltung eines. italien. $d i$ oder da scoglio erklärt.) 


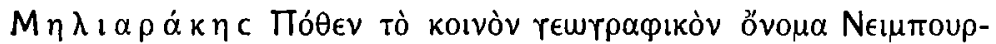

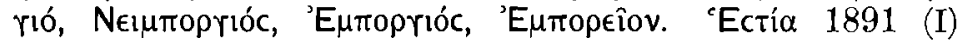
p. $409 \mathrm{ff}$.

(Die Namen sind nach der Ansicht von M. Verstümmelungen von veì̀ $\mu \pi 0 \hat{p}$ ro zu mlat. burgus, haben demnach

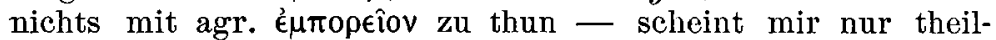
weise richtig.)

Ein Artikel von $\mathrm{J}_{\text {o e }} \mathrm{s}$ (in den Verhandl. der Berl. Ges. f. Anthrop. 1890 p. 210 ff.) über die Etymologie des Wortes Caviar veranlasst Politis in der 'Ectía (Beiblatt) vom 12. August 1890, auf die älteste Fundquelle des Wortes bei Prodromos hinzuweisen; doch leuchtet mir Politis' Etymolo-

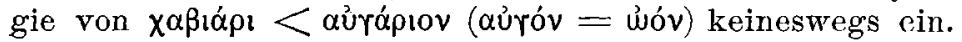

Burys Notiz über vepó The Classical Review V 232 bringt nichts besonderes.

Von sehr zweifelhaftem Wert sind die etymologischen Versuche von Boltz. So hat er seine 'berühmte' Etymologie von ở $\operatorname{cor}$ v (zu ai. gavala!) in der Amsterdamer Zeitschrift ${ }^{`} E \lambda \lambda \alpha$ ác I 1-20 durch eine ebenso abenteuerliche in derselben Zeitschr. II 157-166 ersetzt.

Brauchbarer, wenn auch manches Verkehrte enthaltend und von grosser Weitschweifigkeit, sind desselben Verfassers Lexikologische Beiträge (I. über $\mu \omega \rho \hat{~ e t c . ~ I I . ~} \pi \alpha \lambda \lambda \eta$ kópıov III. - $\pi 0 u \lambda$ oc) im III. Bd. der genannten Zeitschrift. Boltz Verfahren ist unkritisch, weshalb seine Arbeiten nur als Sammlungen von Material einiges Interesse haben.

Zur rein praktischen Einführung in die neugriech. Grammatik haben die beiden letzten Jahre einiges gebracht; es genügt hier auf meinen Aufsatz Die neugriech. Sprache und ihre Erlernung in der Beilage zur Allg. Zeitung No. 181 (6. August 1891) und auf meine Rezension von Sanders Grammatik in Literar. Merkur 1891 No. 9 p. 61 hinzuweisen. In meinem zuerst genannten Aufsatz ging ich besonders auf die prinzipicllen Fragen ein, welche bei der Abfassung einer praktischen neugriechischen Grammatik in betracht gezogen werden müssen. fügen:

Meinem Aufsatz habe ich nachzutragen bezw. hinzuzu-

Manuel de conversation en trente langues par le Dr. Pou ssié avec la collaboration de savants français et étrangers. Paris 1890 (die neugriech. Volkssprache ist von Psichari bearbeitet)

und die

Neugriech. Grammatik von Mitzotakis, herausgegebon vom

Seminar für orientalische Sprachen in Berlin 1891. Beides ist mir bis jetzt nicht zugänglich gewesen. 
Eine grosse wissenschaftliche Grammatik des Vulgärgriechischen ist bekanntlich von Foy schon seit Jahren in Aussicht gestellt worden; ich weiss nicht, wie weit der Plan gediehen ist. Eine historische Grammatik des Neugriechischen mit Einschluss der wichtigsten Dialekte ist freilich bis jetzt kaum zu erwarten, da die streng wissenschaftliche Untersuchung der Einzelfragen sozusagen erst seit wenigen Jahren begonnen hat. Vordcrhand ist die beste Einführung in das Gesammtgebiet der neugriech. Sprachforsehung

W. Meyers Neuausgabe von Portius Grammatica linguae graceae vulgaris. Paris 1889 (s. auch oben).

Im grammatischen Kommentar giebt der verdiente Romanist eine Zusammenstellung des bis heute Erreichten und sucht die wichtigsten grammatischen Fragen im Zusammenhang aufzuhellen und zu erklären; dass manches nur als erster Versueh betrachtet werden kann, ist nicht verwunderlich: das Werk von Heyer zeigt eben, wie viel noch in Neograecis zu thun ist. Vgl. die eingehende Besprechung von

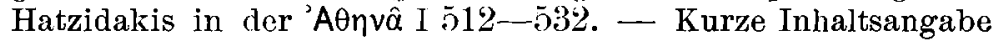
von Flament im 2. Bd. der 'E $E \lambda \alpha \alpha$ c.

Wie weit die ganz vor kurzem erschienene Historische Grammatik der hellenischen Sprache von H. C. Muller wissenschaftlichen Anforderungen genügt, weiss ich nicht, da ich dieselbe noch nicht einsehen konnte.

An dieser Stelle ist nochmals $\mathrm{Krumbachers} G \mathrm{Ge}$ schichte der byzantinischen Literatur zu nennen: kurze Betrachtungen über den Charakter der byzantinischen Schriftund Volkssprache sind an versehiedenen Orten eingestreut; ein besonderer Abschnitt (mit bibliographischen Nachweisen) ist der Charakteristik des Vulgärgriechischen gewidmet (p. 385 -396). In den Vordergrund tritt naturgemäs die Erörterung des litterarischen Verhältnisses zwischen Volks- und Schriftsprache, d. h. der Vertretung und des Kampfes beider Sprachphasen in der mittelgriech. Litteratur.

Die letztgenannte Frage wurde, nur von einem andern Standpunkt aus, von dem hervorragenden Vertreter neugriech. Sprachforschung, dem Griechen $\mathrm{Hatzidak}$ is, in $2 \mathrm{Abhand-}$ lungen erörtert, nämlich :

Zur neugriech. Sprachfrage im I. Bd. der 'E $\lambda \lambda \alpha$ ác und ausführlicher in griechischer Bearbeitung

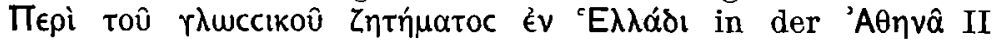
169-235 (sowie separat Athen 1890, Perris. 67 S.).

Dazu meine Rezension im Literar. Centralblatt 1890 Sp. 1677.

Hatzidakis orientirt kurz und präzis über die Geschicke der altgriechischen und die Entstehung der neugriechischen 
Schriftsprache, wobei fortgesetzt auf die Entwicklung der Volkssprache Rücksicht genommen wird. Über die sogen. "Sprachfrage" s. unten.

Die Keime der neugriech. Sprache sind bekanntlich schon im Altertum zu suchen; in der Kovvń finden wir die ersten Ansätze derjenigen Entwicklung des Griechischen, welche in konsequenter Weiterbildung zum Neugriechischen führt. Wir haben daher in unserer Übersicht auch die Untersuchungen über jene Sprachphase zu erwähnen, nämlich: $\mathrm{Simcox}$ The language of the New testament. London 1889.

$226 \mathrm{~S}$. (mir nicht zugänglich).

Rez. von Rendall The Classical Review IV 168 f. und im Athenaeum 1890 (letzteres mir nicht zugänglich).

Schmidt Der Atticismus. 2 Bde. Stuttgart 1887-1889, gleichsam ein altgriech. Gegenstück zur Sprachfrage des modernen Griechenland.

$\mathrm{Buresch}$ rérovav und anderes Vulgärgriechisch. Rhein. Mus. 46 (1891) p. 193-232.

Hellenistische (vulgäre) Formen besonders aus der Bibel und dem sog. "alexandrinischen" Dialekt werden unter Anführung zahlreicher Belege erörtert; die Verhältnisse der Bibelhandschriften in sprachlicher Beziehung finden besonders eingehende Besprechung. Neugriechisches wird nur gestreift.

Um den Sprachcharakter des mittelalterlichen Griechisch hat sich zwischen Hatzidakis und Psichari ein lebhafter, leider oft persönlich geführter Streit entsponnen. Die Frage ist deshalb wichtig, weil sie in letzter Linie auf dic Methode der mittelgr. Sprachforschung abhebt. So enthält denn auch der Aufsatz von

Hatzidakis Zur Geschichte des Mittel- und Neugriechischen, KZ. XXXI 103-15̈31)

vorwiegend Untersuchungen über die Methode, welche wir mittelgriech. Texten gegenüber anzuwenden haben. Ueberzeugend weist $H$. den Mischeharakter der byzantinischen Sprache nach und folgert daraus konsequent, dass eine rein statistische Methode zu keinem Ziel fübrt, dass wir also qualitativ, nicht quantitativ die mittelalterlichen Sprachformen abzuschätzen haben. H. sucht einige Kriterien zu gewinnen, welche uns in byzantinischen Texten die echt volkstümlichen Formen von toten oder monströsen Bildungen scheiden lassen.

Hinsichtlich dor sprachgeschichtlichen Methode in der Erforschung des Neugriechischen hat früher die Frage eine grosse Rolle gespielt, in welchem innern Verhältnis dic altgriech. Dialekte zum Neugriechischen stehen. Nachdem Ha-

1) Angezeigt in der 'E $\lambda \lambda \alpha$ ác II $103 \mathrm{f}$. 
tzidakis seinerzeit die Entstehung des Neugriechischen aus der Koıvń klar erwiesen und damit allen "äolodorischen" Spekulationen ein für alle mal den Garaus gemacht hatte, konnte doch vom neuen Standpunkt aus der Frage wiederum näher getreten werden, ob und wie weit die altgr. Dialekte Spuren im heutigen Griechisch oder in heutigen Dialekten (abgesehen vom Tzakonischen) hinterlassen haben. Die Frage ist zu bejahen, wenn auch jene Spuren verhältnismässig sehr gering sind. Die einigermassen sicheren und bis jetzt bekannten dialektischen Reste sind zusammengestellt von Hatzidakis Zur Abstammungstrage des Neugriechischen. 'E $\mathrm{\lambda \lambda \alpha ́c}$ III 1-5.

Ausser diesen prinzipiellen Erörterungen hat $\mathrm{Hatzi}$ $\mathrm{d} a \mathrm{k}$ is noch spezielle Gebiete der neugriech. Grammatik in folgenden Aufsützen untersucht:

Zum Vokalismus des Neugriechischen. KZ. XXX 35̄--398. Dazu Nachtrag ib. XXXI 153-1 6 .

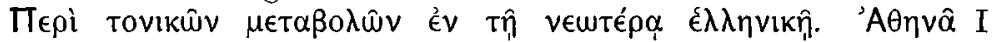
247-287. 481-511.

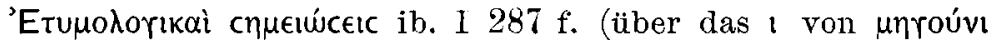

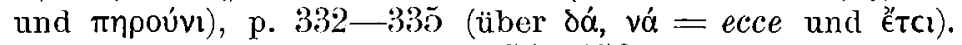

In derselben Zeitschr. II 154-159 Referat eines Vortrags über die neugr. Zahlwörter (welche den Ursprung des Neugriechischen aus der Kotv' erweisen).

II 701-708 Referat eines Vortrag über Geschlechtswechsel im Neugriechischen.

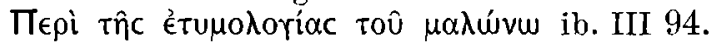

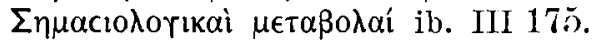

Ich verzichte darauf, an diesem Orte näher auf die Aufsätze des ausgezeichneten Neogräzisten einzugehen, da das Erscheinen eines Buches nahe bevorsteht, worin Hatzidakis seine neugriechischen Forschungen zusammenfasst. Dies wird mir Gelegenheit geben, auf die Bedeutung jener zurückzukommen. Endlich nenne ich noch:

Pavolini Über Dvandva-Komposita im Neugriechisehen.

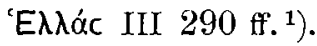

$$
\text { (Schluss folgt.) }
$$

Freiburg i. B., September 1891.

Albert Thumb.

1) Miklosich Über die Einwirkung des Türkischen auf die Grammatik der südosteuropäischen Sprachen. Sitzungsberichte der Wiener Ak. d. Wiss. 120. Bd. behandelt nicht das Neugriechische. 